\title{
Address Decomposition for the Shaping of Multi-dimensional Signal Constellations
}

\author{
A. K. Khandani ${ }^{1}$ and P. Kabal ${ }^{1,2}$ \\ ${ }^{1}$ INRS-Telecommunications, 16 Place du Commerce, Verdun, Canada, H3E 1 H6 \\ ${ }^{2}$ McGill University, 3480 University, Montreal, Canada, H3A 2A7
}

\begin{abstract}
In this work, we introduce an efficient addressing scheme to realize points near to the knee of the tradeoff curves of an optimally shaped constellation. This scheme, called as the address decomposition, is based on decomposing the addressing into a hierarchy of the addressing steps each of a low dimensionality. As the memory size associated with a direct addressing scheme has an exponential growth with the dimensionality, this decomposition of the addressing results in a substantial decrease in the complexity. In this case, by using a memory of a practical size, we can move along a tradeoff curve which is nearly optimum. For example, in a space of dimensionality $N=32$, we use a block of memory of 2.5 kilo-bytes per $N$ dimensions to achieve a shape gain of $\gamma_{s}=0.92 \mathrm{~dB}$ with $\mathrm{CER}_{s}=1.25, \mathrm{PAR}=2.95$. This scheme has no associated computation, is straightforward to implement and is adaptable to the structure of a general coset coding scheme.
\end{abstract}

\section{Introduction}

Consider a discrete source producing one of $M$ equiprobable symbols in each signaling interval. We are going to transmit the sequence of the source symbols to a receiver. Our transmission media is a channel which provides us with a given number of dimensions per signaling interval. To achieve the transmission, we select $M$ points over the channel space. Each source symbol is represented by one of these points. This is called a signal constellation.

The constellation points are usually selected as a finite subset of a regular array of points bounded within a shaping region. In selecting the shaping region, the objective is to minimize the average energy of the constellation. In continuous approximation, the distribution of the constellation points is approximated by a continuous uniform density of points within the shaping region. The reduction in the average energy per two dimensions due to the use of the region $\mathcal{C}$ as the boundary instead of using a hypercube is called the shaping gain of $\mathcal{C}$ and is denoted as, $\gamma_{s}(\mathcal{C})$. The price to be paid for shaping $\left(\gamma_{s}>1\right)$ involves: (i) an increase in the factor CER (Constellation-Expansion-Ratio) which is defined as the ratio of the number of points per two dimensions to the minimum necessary number of points per two dimensions, [1], (ii) an increase in the factor PAR (Peak-to-A verage-power-Ratio) which is defined as the ratio of the peak of energy per two dimensions to the average energy per two dimensions, [1], (iii) an increase in the addressing complexity, where addressing is the assignment of the source symbols to the constellation points ${ }^{2}$.

In general, there exists a tradeoff between $\gamma_{s}$ and CER, and also between $\gamma_{s}$ and PAR. An optimum shaping region is the region which optimizes both of these tradeoffs, [2]. The major problem

\footnotetext{
${ }^{1}$ This work was supported by Natural Sciences and Engineering Research Council of Canada (NSERC)

${ }^{3}$ In a cubic constellation, addressing can be achieved independently along each dimension. This results in low complexity. However, in a shaped constellation independent addressing is not applicable.

$0.7803-0608-2 / 92 / \$ 3.00$ (c) 1992 IEEE
}

associated with the optimum shaping in a high dimensional space is the addressing complexity.

We use continuous approximation to calculate the performance of our schemes. Usually, this results in a lower bound to the actual performance. To justify this, consider a shaping region which is the union of some unity volume Voronoi regions. The centroid of the Voronoi regions are the constellation points. Using the orthogonality principle, the average second moment of the region with respect to the origin is the sum of two terms, namely, the average second moment of the centroids and a second term which is the second moment of a single Voronoi region. In the discrete analysis, the second term is not present.

In all our discussions, we assume that the dimensionality is even and the constellation points are used with equal probability.

\section{A simple example of shaping}

In Figs. 1 and 2, we see two examples of a 64-points 2-D signal constellation from the half integer grid. The one shown in Fig. 1 has a cubic shaping region. Assuming a minimum distance of one, the average energy per dimension of this constellation is equal to 5.25. The constellation in Fig. 2 is obtained by replacing the four points of the highest energy in the cubic constellation with another four points of a lower energy. These are the points marked by the circles. As a result, the average energy has reduced to 5.125 . This corresponds to the shape gain, $\gamma_{s} \simeq 0.1 \mathrm{~dB}$.

The cubic constellation employs 8 points per dimension. This is the minimum necessary number of points per dimension to have 64 points in two dimensions. However, in the shaped constellation, we have employed 10 points per dimension. Assuming that $\mathrm{CER}_{3}$ is measured on a one dimensional basis, this corresponds to, $\mathrm{CER}_{s}=1.25$

The peak of energy per dimension of the cubic and the shaped constellations are equal to 12.25 and 20.25 , respectively. Assuming that PAR is measured on a one dimensional basis, the PAR's are equal to, 2.33 and 3.95 , respectively.

In the cubic constellation, to map the six bits of data to a constellation point, we can use three bits to select a point along one dimension and another three bits to select a point along the other dimension. However, in the shaped constellation, this method is not applicable. In this simple example, we can use a lookup table with 64 memory locations to achieve the addressing. However, for the same number of bits per dimension, namely 3 , in dimensionality 32 , we need a lookup table with $2^{96}$ memory locations which is not practical.

\section{Previous relevant works}

In the work of Wei, [3], shaping is a side effect of the method employed to transmit a nonintegral number of bits per two dimensions. This method provides moderate shaping gain for low 


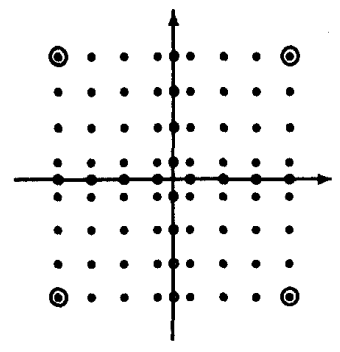

Fig. 1 A 64-points 2-D cubic constellation from half integer grid.

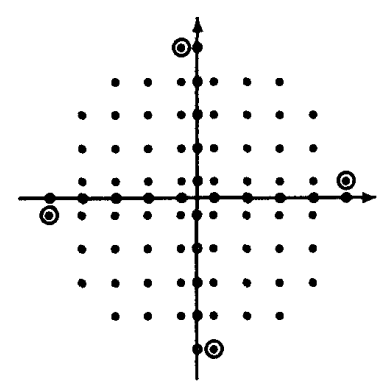

Fig. 2 A 64-points 2-D shaped constellation from half integer grid.

values of CER. The addressing of this method is achieved by a lookup table. Forney and Wei generalize this method under the topic of the generalized cross constellation in [1].

Conway and Sloane in [4] introduced the idea of the Voronoi constellation based on using the Voronoi region of a lattice $\Lambda_{s}$ as the shaping region. In these constellations, the set of the points form a group under vector addition modulo $\Lambda_{s}$. This property is used to achieve the addressing. The complexity of the addressing is that of a linear mapping plus the decoding of the shaping lattice

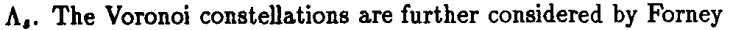
in [5]. The idea of the trellis shaping is introduced in [6]. This is based on using an infinite dimensional Voronoi region, determined by a convolutional code, to shape the constellation.

In [7], Calderbank and Ozarow introduced a shaping method which is directly achieved on the 2-D subspaces. In this method, the 2-D subspaces are partitioned into equal sized shells of increasing average energy. A shaping codes is then used to specify the sequence of the 2-D shells. The shaping code is designed so that the lower energy shells are used more frequently.

Lang and Longstaff in [8] use an addressing scheme which first divides the final constellation into energy shells. Then, a point in a shell is found by successively decomposing the space into lower-dimensional subspaces via generating function techniques. As noted in passing in [8], the same technique can be used for the addressing of a shaped constellation. It should be mentioned that prior to [8], a similar addressing scheme was used by Fischer in [9], to label the points of a vector quantizer with a pyramid quantization region. The addressing scheme of Lang and Longstaff is further discussed and nicely formulated by Kschischang in [10] (see also Kschischang and Pasupathy, [11]). Loria in [12], applies some ideas from a type of structured vector quantizer to the constellation addressing. This results in an addressing scheme similar to that of [9].
In [13], Kschischang and Pasupathy discuss a shaping method which is based on using the 2-D points with nonequal probability (see also [10]). In [14] (see also [15]), Livingston discusses a shaping method in which the 2-D subspaces are partitioned into annular shells of increasing size. In this method, the 2-D shells are used with equal probability inducing a nonuniform distribution on the 2-D points. Recently, Kschischang in [16] discusses the structure of a prefix code which closely approximates the optimum nonuniform probabilities of the 2-D points.

The structure of the region which optimizes the tradeoff between the $\gamma_{s}$ and the CER ${ }_{s}$ and also between the $\gamma_{s}$ and the PAR together with analytical expressions determining the optimum tradeoff is given in [2] (also refer to [17], [18]). Independently, Kschischang in [10] (also refer to Kschischang and Pasupathy, [11]) and Loria in [12] arrive at the same shaping region. References [10] and [11] also give expressions for the optimum tradeoff curves using a different approach than than of [2].

In our work, [19] (also refer to [18]), some practical addressing schemes to achieve (or approximate points) on the optimum tradeoff curves is given. Laroia et al in [20] suggest methods to reduce the computational complexity of the addressing scheme based on the same general idea as in, [8], [12].

In this work we introduce an efficient addressing scheme to realize tradeoff points with negligible suboptimality. This is based on decomposing the addressing into a hierarchy of the addressing steps each of a low dimensionality. As the memory size of a direct addressing scheme has an exponential growth with the space dimensionality, this scheme results in a substantial decrease in the complexity. It should be mentioned that this work is being reported in part at [18], [19].

\section{Shell mapping}

Assume that CER, and PAR are measured on a 2-D basis. In an $N$-D optimally shaped region, $\mathcal{A}_{N}$, a 2-D sphere of radius $R_{2}$, $\mathcal{S}_{2}\left(R_{2}\right)$, is the boundary of the 2-D subspaces and an $N$-D sphere of radius $R_{N}, \mathcal{S}_{N}\left(R_{N}\right)$, is the boundary of the whole space, i.e.,

$$
\begin{aligned}
\mathcal{A}_{N}= & \left\{X_{j}, j=0, \ldots, N-1\right\} \\
: & 0 \leq X_{2 p}^{2}+X_{2 p+1}^{2} \leq R_{2}^{2}, \quad p=0, \ldots, n-1 \\
& 0 \leq \sum_{j=0}^{N-1} X_{j}^{2} \leq R_{N}^{2}, \quad 0 \leq R_{N}^{2} \leq n R_{2}^{2} .
\end{aligned}
$$

By applying the change of variable,

$$
Y_{p}=\left(X_{2 p}^{2}+X_{2 p+1}^{2}\right) / R_{2}^{2}, \quad p=0, \ldots, n-1, n=N / 2,
$$

to (1), the region $\mathcal{A}_{N}$ is mapped to the following $n$-D solid,

$$
\begin{aligned}
\mathcal{T C}_{n}(1, \beta)= & \left\{Y_{p}, p=0, \ldots, n-1\right\} \\
: & 0 \leq Y_{p} \leq 1 \\
& 0 \leq \sum_{p=0}^{n-1} Y_{p} \leq \beta, \quad 0 \leq \beta=R_{N}^{2} / R_{2}^{2} \leq n .
\end{aligned}
$$

This is a hypercube of edge length one truncated within a simplex of edge length $\beta$. We refer to the $N$-D space as the $N$-domain and to the $n$-D space as the $n$-domain

The change of variable in (2) is denoted as the shell mapping. This mapping has an important property that: $A$ uniform density within $\mathcal{A}_{N}$ results in a uniform density within $\mathcal{T C}_{n}$.

Now, consider an optimum shaping region in an $N=N^{\prime} n^{\prime}-\mathrm{D}$ space. Without loss of generality, we assume that the shaping (truncation within the $N$-D sphere) is achieved on the $n^{\prime}$-fold cartesian product of an $N^{\prime}-\mathrm{D}$ unshaped region $\left(\left\{\mathcal{S}_{2}\right\}^{N^{\prime} / 2}\right)$. By the 
use of the shell mapping, each $\left\{\mathcal{S}_{2}\right\}^{N^{\prime} / 2}$ is mapped to an $T \mathcal{C}_{n}(1, n)$ where $n=N^{\prime} / 2$. By applying the change of variable,

$$
Z_{q}=\sum_{p=0}^{n-1} Y_{p}, \quad q=0, \ldots, n^{\prime}-1, \quad n^{\prime}=N / N^{\prime}
$$

each $\mathcal{T C}_{n}$, or equivalently each $\mathcal{A}_{N^{\prime}}$, is mapped to one of the dimensions of an $T \mathcal{C}_{n^{\prime}}$ in a domain denoted as the $n^{\prime}$-domain. Fig. (3) shows an example of such a two-level shell mapped constellation for $N=8, N^{\prime}=4$ and $K=4$.

Each of the $\mathcal{A}_{N}$, regions is partitioned into $K$ energy shells of equal volume. The partitioning of the $\mathcal{A}_{N}$ 's result in equal volume partitions in the $n$-domain produced by the radial hyperplanes, refer to Fig. (3). The partitions of each $\mathcal{A}_{N^{\prime}}$ correspond to the set of the points $\left\{U_{i}, i=0, \ldots, K\right\}$ along the corresponding dimension of the $T \mathcal{C}_{n^{\prime}}$. To achieve the shaping, a subset of the elements in the $n^{\prime}$-fold cartesian product of the set of the partitions is selected. The corresponding addressing is achieved using a lookup table.

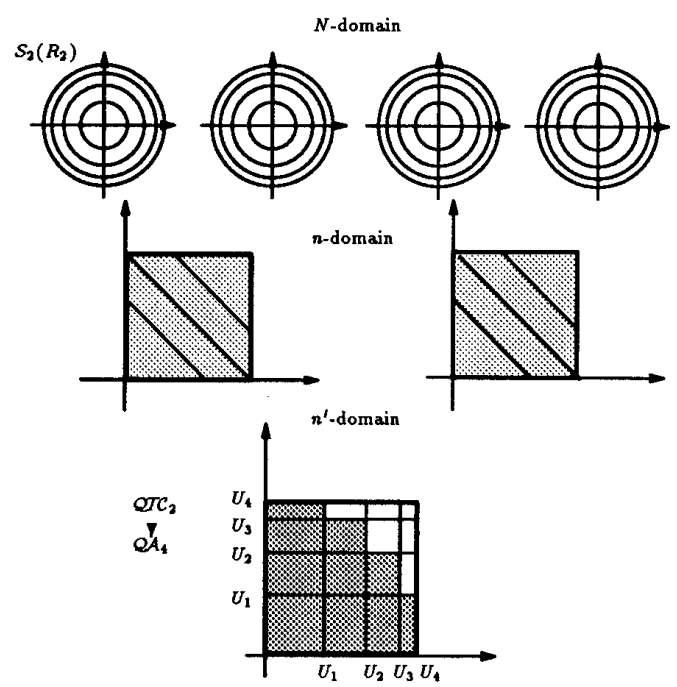

Fig. 3 Example of a two-level shell mapped constellation.

A point $U_{i}$ along a dimension of $\mathcal{T C}_{n^{\prime}}$ corresponds to the region $\mathcal{A}_{N}$, with $\beta=U_{i}$. Using the results of [18], the volume of this region is equal to,

$$
V\left(\mathcal{A}_{N^{\prime}}\right)=\left(\pi R_{2}^{2}\right)^{n} \sum_{k=0}^{\left.\mid U_{j}\right\rfloor}(-1)^{k} C_{n}^{k} \frac{\left(U_{i}-k\right)^{n}}{n !},
$$

where $n=N^{\prime} / 2$. To obtain partitions of equal volume, the points $U_{i}$ should satisfy,

$$
\sum_{k=0}^{\left\lfloor U_{i}\right\rfloor}(-1)^{k} C_{n}^{k} \frac{\left(U_{i}-k\right)^{n}}{n !}=\frac{i}{K} .
$$

Equations (6) can be used to calculate the points $U_{i}$ (for example using a bisection algorithm).

The partitioning of the $N^{\prime}$-D subspaces results in $K^{n^{\prime}}$ equal volume partitions (clusters) in the $N$-domain. Each of these clusters corresponds to a parallelepiped in the $n^{\prime}$-domain. A parallelepiped located at point $\left(U_{I_{0}}, \ldots, U_{I_{n^{\prime}-1}}\right), I_{j \in\left\{0, \ldots, n^{\prime}-1\right\}} \in\{0, \ldots, K\}$ is shown by,

$$
\begin{aligned}
\mathcal{P}\left(U_{I_{0}}, \ldots, U_{I_{n^{\prime}-1}}\right)= & \left\{Z_{q}, q=0, \ldots, n^{\prime}-1\right\} \\
: & U_{I_{0}} \leq Z_{0} \leq U_{I_{0}+1} \\
& \ldots \cdots \cdots \\
& U_{I_{n^{\prime}-1}} \leq Z_{n^{\prime}-1} \leq U_{I_{n^{\prime}-1}+1}
\end{aligned}
$$

Shaping is achieved by selecting $T$ of the $N$-D clusters with the least second moment. In the example of Fig. (3), we have $T=10$. Considering that the first moment of $T \mathcal{C}_{n^{\prime}}$ is proportional to the second moment of the $N$-domain, the selected subset corresponds to the parallelepipeds with the least average first moment. This procedure in fact uses a quantized version of $\mathcal{T C}_{n^{\prime}}$, denoted by $\mathscr{Q T C}_{n^{\prime}}$, as the shaping region in the $n^{\prime}$-domain. The final region is denoted by $\mathcal{Q A}_{N}^{N^{\prime}}(K, T)$.

In a parallelepiped the average first moment is equal to the sum of the average first moments along different dimensions. Using this fact and some other results from [18], it can be shown that,

$$
\begin{gathered}
F_{m}\left(\mathcal{P}\left(U_{I_{0}}, \ldots, U_{I_{k^{\prime}-1}}\right)\right)=\left(\frac{1}{K}\right)^{n^{\prime}-1} \times \\
\sum_{q=0}^{n^{\prime}-1}\left\{\sum_{k=0}^{\left\lfloor U_{I_{q}+1}\right\rfloor}(-1)^{k} C_{n}^{k} \frac{n(\beta-k)^{n+1}+k(n+1)(\beta-k)^{n}}{(n+1) !}-\right. \\
\left.\sum_{k=0}^{\left\lfloor U_{I_{q}}\right\rfloor}(-1)^{k} C_{n}^{k} \frac{(n)(\beta-k)^{n+1}+k(n+1)(\beta-k)^{n}}{(n+1) !}\right\} .
\end{gathered}
$$

This is used to calculate the average first moment of the selected subset of the parallelepipeds, $F_{m}\left(\mathcal{Q T C}_{n^{\prime}}\right)$. The average energy per 2-D of the $N$-domain is equal to,

$$
P_{2}\left(\mathcal{Q A}_{N}^{N^{\prime}}(K, T)\right)=\frac{2 R_{2}^{2}}{N} \frac{K^{n^{\prime}}}{T} F_{m}\left(\mathcal{Q T} \mathcal{C}_{n^{\prime}}\right)
$$

It is easy to show that the volume of $\mathcal{Q} A_{N}^{N^{\prime}}$ is equal to,

$$
V\left(\mathcal{Q A}_{N}^{N^{\prime}}(K, T)\right)=\left(\pi R_{2}^{2}\right)^{N / 2} \frac{T}{K^{n^{\prime}}} .
$$

Equations (9) and (10) can be used to calculate the tradeoff. The final results for $n^{\prime}=2$ is shown in Fig. (4). The optimum tradeoff curves $(K=\infty)$ are extracted from [2].

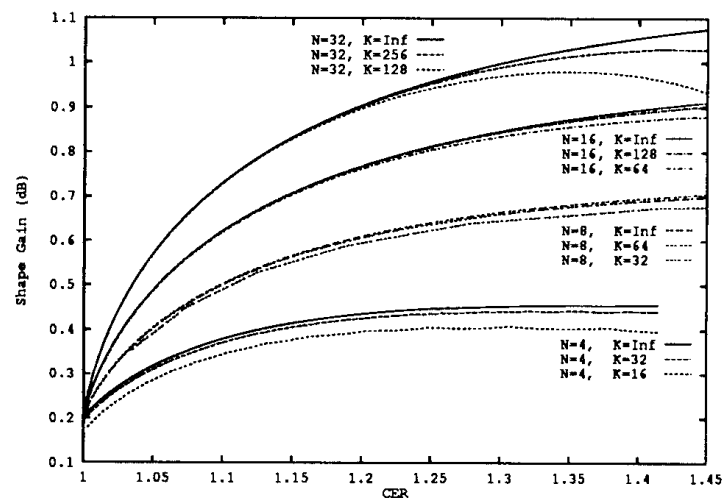

Fig. 4 Tradeoff between $\mathrm{CER}_{s}$ and $\gamma_{s}$ using a finite number of the energy shells in the $N / 2-\mathrm{D}$ subspaces. 


\section{Address decomposition}

Referring to Fig. (4), for moderate values of CER, in an $N$-D space, one can closely approximate the optimum tradeoff using a small number of equi-volume energy shells per $N / 2-D$ subspaces. A similar effect over dimensionality two was observed for the first time in [7]. This property allows us to decompose the addressing of a constellation into some intermediate steps achieved on the 2 -fold cartesian product of a set with low cardinality. We call this method as the address decomposition.

For a dimensionality $N=2^{*}$, this results in $u-1$ addressing steps. The $i$ 'th step, $i \in[1, u-1]$, is achieved on the $2^{i}$-D subspaces and results in dimensionality $2^{i+1}$. We assume that the subspaces involved in the $i$ 'th step are partitioned into $K_{i}=2^{k_{i}}$ shells. Referring to Fig. (4), we select $\left\{k_{i}, i=1, \ldots\right\}=\{6,6,7,8, \ldots\}$. The $i$ 'th addressing step requires a memory with $2 k_{i} \times 2^{2 k_{i}}$ bits. The last step requires $2 k_{i} \times 2^{2 k_{i}-r}$. bits, where $r_{s}=(N / 2) \log _{2} C E R_{s}$. An upper bound to total memory size, $M_{t}$, is obtained by setting $r_{s}=0,\left(\mathrm{CER}_{s}=1\right)$.

Figure (5) shows the final tradeoff curves. The optimum tradeoff curves are extracted from [2]. It is seen that the overall suboptimality is negligible.

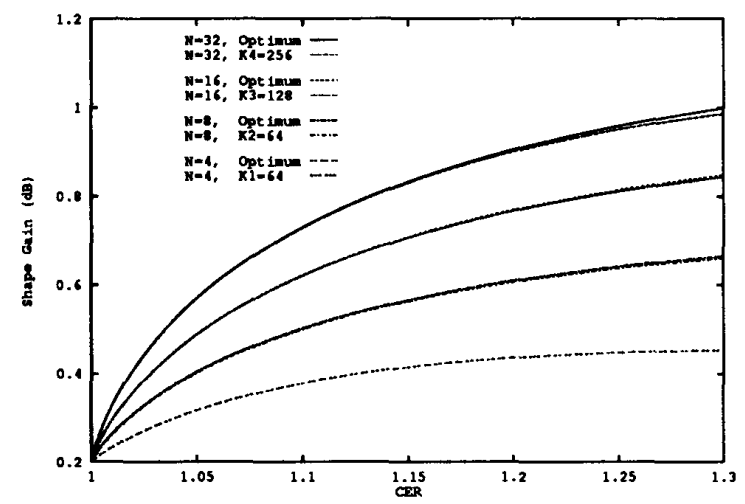

Fig. 5 Tradeoff between CER, and $\gamma_{s}$ using the address decomposition method.

The introduced addressing scheme has some similarities with the scheme of [8]. The key point to the scheme of [8] is that the weight distribution of the integer lattice (or more generally a lattice which is equal to the cartesian product of some lower dimensional sublattices) is equal to the convolution of the weight distributions in its subspaces. This fact is used in [8] to successively decompose the addressing into lower-dimensional subspaces via generating function techniques. The major difference is the here, by aggregating the energy shells in higher dimensional spaces, we have been able to avoid the computation of the convolutions. This substantially reduces the complexity while the suboptimality is negligible.

It should be mentioned that by selecting smaller values for the $k_{i}$ 's, one can further decrease the size of the memory at the price of some performance degradation. It is specifically appropriate to use a small number of partitions in the first $u-2$ addressing stages, and a large number at the last stage. This is due to the facts that: (i) shaping is achieved at the last stage and, consequently, a higher resolution at this stage has a more important effect on the overall performance, (ii) even for a large $k_{v-1}$, due to the subtraction of $r_{s}$, the addressing of the last stage does not need a large lookup table. Table (1) shows some examples of the performance and complexity of the proposed scheme.

\begin{tabular}{cccccc}
\hline$N$ & CER $_{s}$ & PAR & $\gamma_{s} \mathrm{~dB}$ & $M_{t}$ & $\left\{k_{i}, i=1, \ldots\right\}$ \\
\hline 16 & 1.25 & 2.86 & $0.79(0.81)$ & $1.5 \mathrm{k}$ & $\{4,4,6\}$ \\
16 & 1.35 & 3.12 & $0.84(0.87)$ & $1.0 \mathrm{k}$ & $\{4,4,6\}$ \\
16 & 1.50 & 3.52 & $0.90(0.92)$ & $2.6 \mathrm{k}$ & $\{4,5,7\}$ \\
\hline 32 & 1.25 & 2.95 & $0.92(0.96)$ & $2.5 \mathrm{k}$ & $\{4,4,5,7\}$ \\
32 & 1.35 & 3.21 & $0.95(1.03)$ & $2.0 \mathrm{k}$ & $\{4,4,5,7\}$ \\
32 & 1.35 & 3.25 & $1.00(1.03)$ & $12 \mathrm{k}$ & $\{5,5,6,8\}$ \\
\hline
\end{tabular}

Table 1 Parameters of the point achieved using the addressing decomposition method. The values inside parenthesis are the optimum $\gamma_{s}$. Column $M_{t}$ is memory size in bytes ( $\left.8 \mathrm{bits}\right) / N-\mathrm{D}$ (no computation).

\section{Comparison with other techniques}

In the following, we compare our addressing schemes with the schemes of [5], [6] [7], and [20].

As we are essentially able to closely approximate any point up to the knee of the optimum tradeoff curves, in Table (2), we have compared some of the values obtained in [5] and [7] with the optimum values calculated in [2].

\begin{tabular}{ccccccc}
\hline & \multicolumn{3}{c}{$\bar{V} C,[5]$} & \multicolumn{3}{c}{$C / O,[7]$} \\
$N$ & $\gamma_{s}$ & CER, & PAR & $\gamma_{s}$ & CER, & PAR \\
\hline 4 & 0.37 & $1.41(1.09)$ & $4.62(2.27)$ & - & - & - \\
8 & 0.65 & $2.00(1.26)$ & $6.98(2.81)$ & 0.60 & $1.27(1.19)$ & $2.80(2.61)$ \\
12 & 0.75 & $3.00(1.26)$ & $8.24(2.86)$ & 0.63 & $1.55(1.13)$ & $3.42(2.51)$ \\
16 & 0.81 & $1.54(1.24)$ & $5.58(2.88)$ & 0.69 & $1.45(1.14)$ & $3.02(2.55)$ \\
24 & 1.03 & $5.20(1.50)$ & $15.2(3.67)$ & 0.80 & $1.50(1.16)$ & $3.46(2.67)$ \\
32 & 0.85 & $1.35(1.16)$ & $4.96(2.70)$ & 0.86 & $1.46(1.17)$ & $3.40(2.72)$ \\
\hline
\end{tabular}

Table 2 Comparison between the the Voronoi constellations $(V C)$ and the Calderbank, Ozarow method $(C / O)$ with the optimum constellations, the values in the parenthesis are the optimum values of CER, PAR for the given $\gamma_{s}$.

It should be mentioned that by applying the peak constraint technique, it is possible to modify the Voronoi constellation in such a way that the 2-D points outside a circle of selected radius are not allowed, [5]. This constraint can be applied to the minimum distance decoder, [21], of the lattice. Such a modification, to some extent, improves the performance of a Voronoi constellation. For example, our simulation results show that for the lattice $E_{8}$, one can achieve almost all the shape gain given in Table (2) but with $\mathrm{CER}_{s}=1.7$ and PAR $=4$ instead of $\mathrm{CER}_{s}=2$ and PAR $=6.98$. It should be mentioned that most probably for the higher dimensional lattices (like $\Lambda_{24}$ ), the improvement will be more pronounced.

As a more detailed comparison, a four state trellis diagram of [6] (in conjunction with the peak constraint technique) achieves $\gamma_{s}=0.97 \mathrm{~dB}, \mathrm{CER}_{s}=1.5, \mathrm{PAR}=3.75$. For $N=32$, a twolevel shaping code of [7] achieves, $\gamma_{s}=0.86 \mathrm{~dB}, \mathrm{CER}_{s}=1.46$, $\mathrm{PAR}=3.40$. In $[20]$, an example for $N=64$ is given which needs 15 multiply-adds per 2-D, together with a total memory of 1.5 kilo-bytes/ $N-\mathrm{D}$, to achieve a tradeoff point with $\mathrm{CER}_{2}=1.5$ near to the optimum curve (the optimum $\gamma_{\mathbf{s}}$ for $N=64, \mathrm{CER}_{s}=1.5$ is equal to, $1.2 \mathrm{~dB}$ ).

Table (1) contains some examples of the performance of our scheme. It seems that our method, which has no associated computation, is easier to implement. 


\section{Application to lattice vector quantization}

The ideas presented here for the addressing of the $\mathcal{T C}_{n} / \mathcal{A}_{N}$ regions can be naturally used in conjunction with a lattice vector quantizer with a pyramid/spherical shaping region. Generalization to the case of a region with nonequal values of power along different dimensions (for example in the case of the transform coding) and/or to the case that the quantization region is composed of a set of nested regions with nonequal densities in different layers, [22], [23], is straightforward.

\section{Summary and conclusions}

We discussed an addressing scheme based on partitioning the constellation into clusters of equal volume and then selecting a subset of the clusters with the lowest average energy. The addressing of this subset is achjeved by a lookup table. By using the address decomposition method, we have substantially decreased the size of the lookup table while the suboptimality is negligible. The key point is that for moderate values of CER, in an $N$-D space, one can closely approximate the optimum tradeoff using a small number of equi-volume energy shells per $N / 2-\mathrm{D}$ subspaces. This method uses a modest amount of memory, has no associated computation, and can be easily used in conjunction with a general coset coding scheme. It is also possible to achieve the shaping in higher dimensional spaces and use its lower dimensional subspaces for the channel coding. As an example of performance, in an $N=32-\mathrm{D}$ space, by increasing the number of the 2-D points by $25 \%$ and using 2.5 kilo-bytes of memory, we realize a shaping gain of $0.92 \mathrm{~dB}$. It seems that after the initial coding gain associated the Ungerboeck's schemes, [24], or with the Wei's schemes, [3], this is the easiest way to obtain higher gains.

\section{References}

[1] G. D. Forney, Jr. and L. F. Wei, "Multidimensional constellations-Part I: Introduction, figures of merit, and generalized cross constellations," IEEE J. Select. Areas Commun., vol. SAC-7, pp. 877-892, August 1989.

[2] A. K. Khandani and P. Kabal, "Shaping multi-dimensional signal spaces-Part I: optimum shaping, shell mapping," submitted to IEEE Trans. Inform. Theory, July 1991, revised March 1992.

[3] L. F. Wei, "Trellis coded modulation with multidimensional constellations," IEEE Trans. Inform. Theory, vol. IT-33, pp. 483-501, July 1987.

[4] J. H. Conway and N. J. A. Sloane, "A fast encoding method for lattice codes and quantizers," IEEE Trans. Inform. Theory, vol. IT-31, pp. 106-109, January 1985.

[5] G. D. Forney, Jr., "Multidimensional constellations-Part II: Voronoi constellations," IEEE J. Select. Areas Commun., vol. SAC-7, pp. 941-958, August 1989.

[6] G. D. Forney, "Trellis shaping," IEEE Trans. Inform. Theory, vol. IT-38, pp. 281-300, March 1992.

[7] A. R. Calderbank and L. H. Ozarow, "Nonequiprobable signaling on the Gaussian channel," IEEE Trans. Inform. Theory, vol. IT-36, pp. 726-740, July 1990.

[8] G. R. Lang and F. M. Longstaff, "A leech lattice modem," IEEE J. Select. Areas Commun., vol. SAC-7, pp. 968-973, August 1989.
[9] T. R. Fischer, "A pyramid vector quantizer," IEEE Trans. Inform. Theory, vol. IT-32, pp. 568-583, July 1986.

[10] F. R. Kschischang, Shaping and coding gain criteria in signal constellation design, Ph.D. Dissertation, Toronto Univ., June 1991.

[11] F. R. Kschischang and S. Pasupathy, "Optimal shaping properties of the truncated polydisc," Submitted to IEEE Trans. Inform. Theory, 1992.

[12] R. Laroja, "On optimal shaping of multidimensional constellations - an alternative approach to lattice-bounded (voronoi) constellations," Submitted to IEEE Trans. Inform. Theory, November 1991.

[13] F. R. Kschischang and S. Pasupathy, "Optimal nonuniform signaling for Gaussian channels," Submitted to IEEE Trans. Inform. Theory, November 1990.

[14] J. R. Livingston, "Shaping using variable-size regions," IEEE Trans. Inform. Theory, vol. IT-38, pp. 1347-1353, July 1992.

[15] J. N. Livingston, "Shaping gains using non-equiprobable signaling," Proc. IEEE Global Telecommunications Conference, pp. 1994-1998, Dec 1990.

[16] F. R. Kschischang, "Huffman codes for shaping gain," 16th Biennial Symposium on Communications, pp. 79-82, Kingston, Ontario, 27-29 May 1992.

[17] A. K. Khandani and P. Kabal, "Shaping multidimensional signal constellations," IEEE Int. Symp. Inform. Theory, p. 4, June 1991, Budapest, Hungary.

[18] A. K. Khandani Shaping multi-dimensional signal spaces, Ph.D. Dissertation, McGill Univ., March 1992.

[19] A. K. Khandani and P. Kabal, "Shaping multi-dimensional signal spaces-Part II: shell-addressed constellations," submitted to IEEE Trans. Inform. Theory, July 1991, revised March 1992.

[20] R. Laroia, N. Farvardin and S. Tretter, "On SVQ shaping of multidimensional constellations - high-rate largedimensional constellations," Twenty-sixth annual conference on information sciences and systems, Princeton, NJ, 19-20 March 1992, (to appear in the conference proceedings).

[21] G. D. Forney, "Coset codes-Part II: Binary lattices and related codes," IEEE Trans. Inform. Theory, vol. IT-34, pp. 1152-1187, September 1988.

[22] D. G. Jeong and J. D. Gibson, "Image coding with uniform and piecewise-uniform vector quantizers," Proc. IEEE Global Telecommunications Conference, pp. 321-324, Dec 1991.

[23] D. G. Jeong and J. D. Gibson, "Uniform and piecewise uniform lattice vector quantization for memoryless Gaussian and laplacian sources," submitted to IEEE Trans. Inform. Theory, Jan 1991, revised March 1992.

[24] G. Ungerboeck, "Channel coding with multilevel/phase signals," IEEE Trans. Inform. Theory, vol. IT-28, pp. 55-67, 1982. 